\title{
Water Reverberation Travel Time Analysis Acquired Using Multi-Depth Streamers
}

\author{
Po-Yen Tseng ${ }^{1}$, Young-Fo Chang ${ }^{1, *}$, Chih-Hsiung Chang ${ }^{2,3}$, and Ruey-Chyuan Shih ${ }^{1}$ \\ ${ }^{1}$ Institute of Seismology, National Chung Cheng University, Chiayi County, Taiwan, R.O.C. \\ ${ }^{2}$ General Education Center, National Chiayi University, Chiayi City, Taiwan, R.O.C. \\ ${ }^{3}$ Center of Energy Research \& Sensor Technology, National Chiayi University, Chiayi City, Taiwan, R.O.C.
}

Received 6 May 2015, revised 2 February 2016, accepted 18 February 2016

\begin{abstract}
Ghost reflections and water reverberations are major and inevitable seismic noises in marine seismic exploration. More recently, new receiver deployment techniques at different sea depths for signal-to-noise ratio (SNR) enhancement are developing. The reverberation characteristics must be known before applying the reverberation attenuation methods. This paper studies the characteristics of reverberations acquired using multi-depth streamers by analyzing the seismic ray path geometry and the scaled physical model data. The study results show that the primary reflection waveforms and reverberations are broadened with an increase in offset. The reverberation waveforms are quite different from those of primary reflections due to the wide-angle reflection. Under shallow water and small spread approximation, new arrival time equations for the primary reflections and reverberations are derived and fit the scaled physical model data very well. The depth-arrival time relationships of the primary reflections and reverberations in the common-source vertical-array gather are linear but their depth-arrival time relationship slopes are different. The primary reflection slopes are the same for different common-source vertical-array offsets but the reverberation slopes increase with offsets.
\end{abstract}

Key words: Reverberation, Multi-depth streamers

Citation: Tseng, P. Y., Y.F. Chang, C. H. Chang, and R. C. Shih, 2016: Water reverberation travel time analysis acquired using multi-depth streamers. Terr. Atmos. Ocean. Sci., 27, 791-798, doi: 10.3319/TAO.2016.02.18.01(T)

\section{INTRODUCTION}

In marine seismic exploration the hydrophone streamer is towed at a fixed depth in the water behind the survey vessel to collect seismic data. The major and inevitable seismic noises encountered in the acquired seismic data are the ghost reflections and water reverberations (Backus 1959; Robinson and Treitel 2000). Ghost reflections originate from a strong reflection interface existing above the shot level (Van Melle and Weatherburn 1953); for example, the bottom of a weathering zone and the sea surface for land and marine seismic shooting, respectively. The seismic waves can propagate downwards and upwards from the source. The upward propagating seismic wave reflects at the strong reflection interface, then goes down into the earth, like a "ghost" that immediately follows the downward seismic wave. Therefore, ghost reflections arrive immediately after

\footnotetext{
* Corresponding author

E-mail:seichyo@ccu.edu.tw
}

primary reflections. Reverberations are the repeated reflections of seismic waves between the sea surface and seabed, which are also known as seismic resonance or ringing. They are one of several multiple reflections (multiples). These repeated reflections are trapped in the water layer but they can also transmit into the seabed and propagate into the earth, resulting in a "multi-source" marine seismic exploration. Therefore, reverberations not only contaminate the reflection waves but also allow the reflection wave to appear periodically in the seismic section (Backus 1959; Robinson and Treitel 2000).

The dual-sensor data (geophone and hydrophone) acquired using an ocean-bottom cable was recently used to separate the up- and down-reflecting waves, and consequently applied to attenuate the ghost reflections (Canales and Bell 1996) and multiples (Van Borselen et al. 2011). Ghost reflections can strongly modulate the spectrum and reduce the energy at notch frequencies. New streamer 
technology has been developed for deploying multi-depth streamers in a vertical plane with a sufficient accuracy and the data acquired by over/under streamers could be used to eliminate the ghost reflections, thus extended the seismic bandwidth (Monk 1990; Moldoveanu et al. 2007; Ferber 2008; Özdemir et al. 2008). Furthermore, reverberations can be rejected using a mode forming technique with a vertical line array (Arvelo and Zabal 1997).

Since more multi-depth receivers are being used to detect seismic waves for signal-to-noise ratio (SNR) enhancement, the characteristics of the reverberations must be known before applying reverberation attenuation methods. This paper focuses on studying the characteristics of reverberations acquired using multi-depth streamers, especially for their travel times. A geometric analysis of the primary reflection and reverberation ray paths is given to derive the new equations about their offset-arrival time and depth-arrival time relationships. In order to exclude the unknown characteristics of the Earth's environment, the expensive and difficult field test, physical modeling (Ebrom and McDonald 1994) was employed to acquire the scaled seismic data. The scaled seismic data were then analyzed and compared with the theoretical arrival times of the water-bottom reflections, the primary reflections and the reverberations and those predicted by the new derived equations to characterize the reverberation properties acquired by the multidepth streamers.

\section{TRAVEL TIMES OF THE PRIMARY REFLECTIONS AND REVERBERATIONS}

\subsection{Primary Reflections}

\subsubsection{Common-Source Horizontal-Array Gather}

Consider an exploration environment, including a horizontal stratum covered by shallow water (shown in Fig. 1), where $x$ is the offset, $h_{1}$ is the water depth and $h_{2}$ is the thickness of the stratum. The $\mathrm{P}$-wave velocities of the water and stratum are $V_{w}$ and $V_{\text {stra }}$, respectively. For a small-spread (offsets are small compared with the depth of target) and shallow water condition, $h_{2} \gg h_{1}$ and $h_{1}+h_{2}>>x$, the offset-travel time equation of the primary reflections reflected from the bottom of the stratum can be approximated by (Taner and Koehler 1969; Kleyn 1982):

$t(x)^{2}=\left(t_{01}+t_{02}\right)^{2}+\frac{(x)^{2}}{V_{r m s}^{2}}$

where $t_{01}=2 h_{1} / V_{w}, t_{02}=2 h_{2} / V_{\text {stra }}$ and $V_{\text {rms }}$ is the rootmean-square velocity of the water and stratum.

In such an exploration condition the ray paths of the primary reflections will be parallel to each other and they will have the same length in the water layer. A virtual source and a virtual receiver located on the water-stratum interface can then be imaged. The offset-travel time relationship of seismic waves reflected from the stratum bottom for the virtual source and virtual receiver can be expressed as

$t^{\prime}(x)^{2}=t_{02}^{2}+\frac{\left(x^{\prime}\right)^{2}}{V_{\text {stra }}^{2}}$

where $x^{\prime}=x-2 d x$ and $t^{\prime}=t-2\left(l / V_{w}\right)$. The horizontal distance between the source and virtual source (or the horizontal distance between the receiver and virtual receiver) is $d x, l$ is the propagation distance of the primary reflections in the water layer. This equation is a transformation of the hyperbolic time-distance equation.

\subsubsection{Common-Source Vertical-Array Gather}

The same two-layer model but with horizontal distance zoomed in 1.5 times is shown in Fig. 2. The depth-travel time equation for the primary reflection in a common-source vertical-array gather is

$t(z)=\frac{\sqrt{h_{1}^{2}+x_{1}^{2}}+\sqrt{\left(h_{1}-z\right)^{2}+x_{3}^{2}}}{V_{w}}+\frac{2 \sqrt{h_{2}^{2}+x_{2}^{2}}}{V_{\text {stra }}}$

where $x_{1}$ is the horizontal distance between the source and refracted point beneath the source, $x_{2}$ is the half distance between two refracted points on the water-stratum interface and $x_{3}$ is the horizontal distance between the receiver and refracted point beneath the receiver. $x_{1}=\tan \theta_{1} \cdot h_{1}, x_{2}=\tan \theta_{2} \cdot h_{2}$, and $x_{3}=\tan \theta_{1} \cdot\left(h_{1}-z\right)$, then $x=x_{1}+2 x_{2}+x_{3} . \theta_{1}$ is the incidence angle of the ray and $\theta_{2}$ is the refraction angle. According to Snell's law, $\sin \left(\theta_{1}\right) / V_{w}=\sin \left(\theta_{2}\right) / V_{\text {stra }}$.

In a condition of $h_{2}>h_{1}, h_{1}+h_{2}>>x$, and $V_{\text {stra }}>V_{w}$, all ray paths of the primary reflections in the water layer will be parallel to each other and the difference in ray incidence angles can be ignored. The difference in primary reflection travel time detected by receivers at adjacent depths is

$t_{1}-t_{2}=\Delta t=\frac{\Delta l}{V_{w}}$

where $\Delta l=\frac{\Delta z}{\cos \theta_{1}}$, which is the difference in travel distance for the primary reflections in the water layer detected by two adjacent receivers at different depths. The continuous derivative of $t(z)$ with respect to the water depth $z$ is

$\frac{d t(z)}{d z}=-\frac{\Delta t}{\Delta z}=-\frac{1}{V_{w}} \cos \theta_{1}$

For the primary reflections in a common-source verticalarray gather $\theta_{1}$ can be considered as a constant. The deptharrival time relationship of the primary reflection can then 


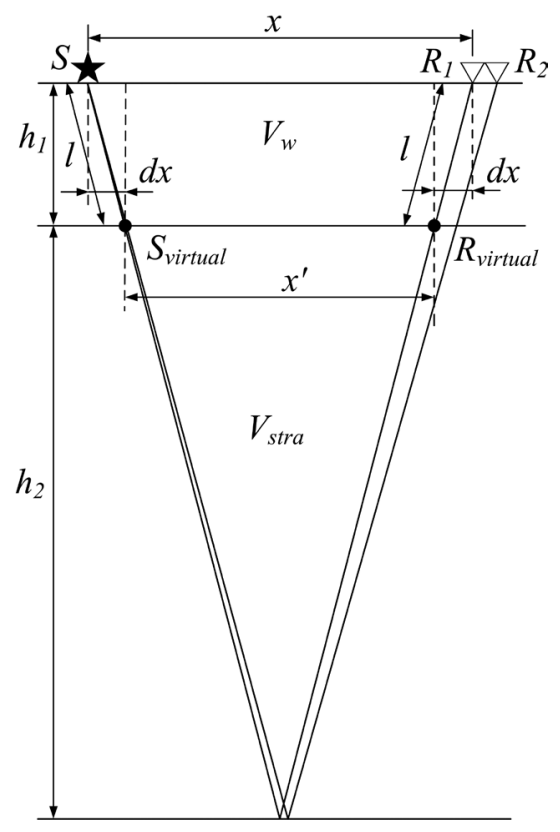

Fig. 1. Ray paths of the primary reflections for a common-source horizontal-array gather in a shallow water environment. $\star$ represents the source, $\nabla$ is the receiver, $x$ is the offset between the source and the receiver, $h_{1}$ is the water depth, and $h_{2}$ is the thickness of the stratum. The P-wave velocities of the water and stratum are $V_{w}$ and $V_{\text {stra }}$, respectively. $l$ is the propagation distance of the primary reflections in the water layer. $S_{\text {virtual }}$ and $R_{\text {virtual }}$ are the virtual source and virtual receiver on the water-stratum interface. $x^{\prime}$ is the offset between the virtual source and the virtual receiver. $d x$ is the horizontal distance between the actual source and the virtual source (or between the actual receiver and virtual receiver).

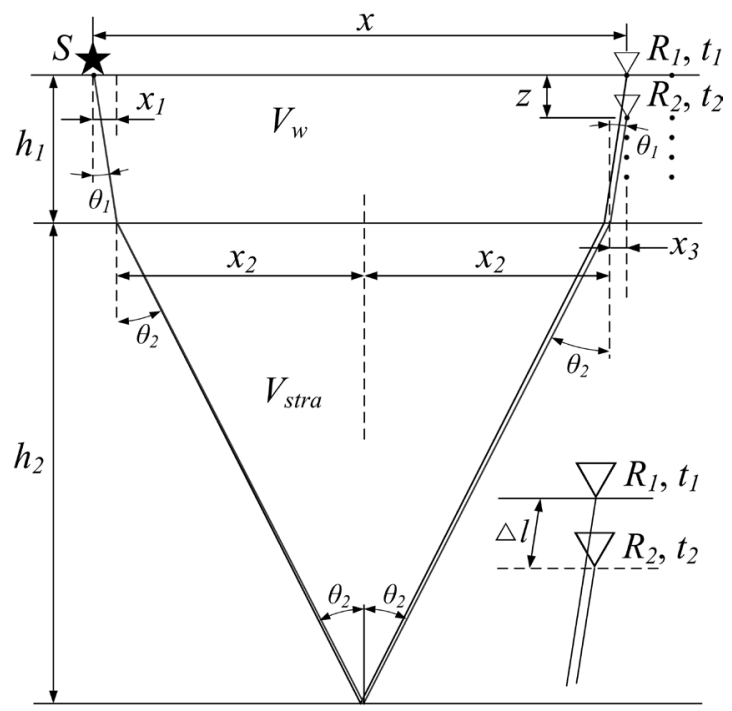

Fig. 2. Ray paths of the primary reflections detected at the water surface and the water depth $z$. The horizontal distance is zoomed in 1.5 times. $\theta_{1}$ is the incidence angle and $\theta_{2}$ is the refraction angle. $\Delta l$ is the difference in travel distance for primary reflections in the water layer detected by receivers at two different depths. $x_{1}$ is the horizontal distance between the source and the refracted point beneath the source, $x_{2}$ is the half distance between two refracted points on the water-stratum interface, and $x_{3}$ is the horizontal distance between the receiver and the refracted point beneath the receiver. be considered as linear under the small spread and shallow water assumption.

\subsection{Reverberation}

\subsubsection{Common-Source Horizontal-Array Gather}

In the common-source horizontal-array gather deployed along the water surface, the ray path of the first-order multireflection of the reverberations is shown in Fig. 3. Under such conditions the ray can be imagined as radiating from the virtual source and the offset-travel time equation is

$t(x)^{2}=\left(4 t_{01}\right)^{2}+\frac{(x)^{2}}{V_{w}^{2}}$

For the $n$-th order multi-reflection of the reverberations the offset-travel time equation is

$$
t(x)^{2}=\left(2 n t_{01}\right)^{2}+\frac{(x)^{2}}{V_{w}^{2}}
$$

\subsubsection{Common-Source Vertical-Array Gather}

In the common-source vertical-array gather the depthtravel time equation of the first-order multi-reflection of the reverberations radiated from the virtual source is

$t(z)=\frac{\sqrt{x^{2}+\left(4 h_{1}-z\right)^{2}}}{V_{w}}$

Expanding Eq. (8) gives

$t(z)=\frac{\sqrt{\left[x^{2}+\left(4 h_{1}\right)^{2}-8 h_{1} z+z^{2}\right] \cdot \frac{x^{2}+\left(4 h_{1}\right)^{2}}{x^{2}+\left(4 h_{1}\right)^{2}}}}{V_{w}}$ Since $h_{1}>z, x^{2}+\left(4 h_{1}\right)^{2}>>z^{2}$ then $\frac{z^{2}}{x^{2}+\left(4 h_{1}\right)^{2}} \approx 0$.
Equation (9) can be approximated as

$t(z)=\frac{\sqrt{1-\frac{8 h_{1} z}{x^{2}+\left(4 h_{1}\right)^{2}}}}{V_{w}} \times \frac{\sqrt{x^{2}+\left(4 h_{1}\right)^{2}}}{1}$

Since $x^{2}+\left(4 h_{1}\right)^{2}>>8 h_{1} z, \sqrt{1-\left\{8 h_{1} z /\left[x^{2}+\left(4 h_{1}\right)^{2}\right]\right\}}$ can be expanded by the Taylor series. After eliminating the highorder term of the Taylor series, Eq. (10) becomes

$t(z)=\frac{1-\frac{1}{2} \cdot \frac{8 h_{1} z}{x^{2}+\left(4 h_{1}\right)^{2}}}{V_{w}} \times \frac{\sqrt{x^{2}+\left(4 h_{1}\right)^{2}}}{1}$ 


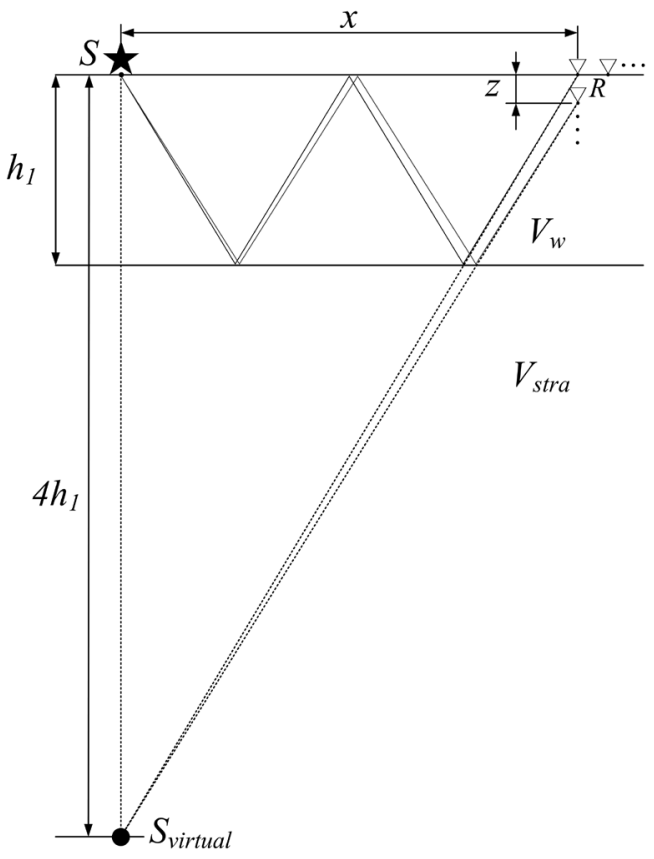

Fig. 3. Ray paths of first-order multi-reflection for reverberations detected at the water surface and water depth $z$.

Arranging Eq. (11) yields

$t(z)=\frac{\sqrt{x^{2}+\left(4 h_{1}\right)^{2}}}{V_{w}}-\frac{4 h_{1}}{V_{w} \sqrt{x^{2}+\left(4 h_{1}\right)^{2}}} z$

Equation (12) shows that the depth-travel time relationship of the reverberations is linear under the small spread and shallow water assumption. Their slope is a function of the offset $(x)$. For $n$-th order multi-reflection of the reverberations the depth-travel time equation is

$t(z)=\frac{\sqrt{x^{2}+\left(2 n h_{1}\right)^{2}}}{V_{w}}-\frac{2 n h_{1}}{V_{w} \sqrt{x^{2}+\left(2 n h_{1}\right)^{2}}} z$

\section{PHYSICAL EXPERIMENTS}

To evaluate the primary reflection and reverberation arrival time equations and characterize the reverberation properties a seismic physical modeling simulation was carried out. A Panametrics V103 transducer with a $1 \mathrm{MHz}$ center frequency, $6.8^{\circ}$ beam width and $13 \mathrm{~mm}$ diameter was adopted to serve as the source. A Panametrics A133S transducer with a $2.25 \mathrm{MHz}$ center frequency and $6 \mathrm{~mm}$ diameter was adopted to serve as the receiver. The pulser/receiver (Panametrics 5058PR) was employed to excite the source-transducer, synchronize (SYN) the digital oscilloscope and receive the ultrasonic signal retrieved from the receiver-transducer. The retrieved signal was then sent to and displayed on a digital oscilloscope (Tektronix TDS 5032B). The digitized radiofrequency (RF) signal was downloaded from the digital oscilloscope via a general-purpose interface bus (GPIB) and IEEE-488 communication to the personal computer. Once the digitized RF signal was retrieved, the receiver-transducer was automatically moved to the next location by the scanning system. The digitized RF signal sample interval and record length used in these experiments were $40 \mathrm{~ns}$ and $400 \mu \mathrm{s}$, respectively. The laboratory setup is shown in Fig. 4.

To simulate the marine seismic exploration environment a copper block $40 \mathrm{~cm} \times 15 \mathrm{~cm} \times 23 \mathrm{~cm}$ was soaked in water at a depth of $7 \mathrm{~cm}$ to serve as a single horizontal stratum beneath the water. The P-wave velocities of the water and copper are 1500 and $4700 \mathrm{~m} \mathrm{~s}^{-1}$, respectively. The source was fixed under the water surface to model a common-source reflection operation. A configuration of 9 streamers with 14 receivers, each located at different water depths from 0 to $4 \mathrm{~cm}$ with an increase of $0.5 \mathrm{~cm}$ was simulated. The lateral near offset between the source and receiver was $2.5 \mathrm{~cm}$. The horizontal interval between the receivers was $1.5 \mathrm{~cm}$. A scaling factor between the laboratory and field was 1:10000 used in both the time and space dimensions. Reverberations usually possess larger amplitude than the other signals in the marine seismic data and this setting allows the primary reflections to be purposely interfered with by reverberations. Therefore, we can easily compare the amplitudes between the primary reflections and reverberations.

\section{RESULTS AND DISCUSSIONS}

This study simulated the deployment of several streamers at different water depths to record multiple seismic waves simultaneously. One of the common-source survey lines measured along the water surface is shown in Fig. 5. As can be seen, both the time and spatial dimensions are multiplied by the scaling factor of 10000 for mapping to the field scale. Automatic gain control (AGC) was applied to the acquired data with a 0.25 second AGC window length. Three dominant events can be observed in the figure. The first event is the top water-copper interface reflections, which simulate the water-bottom reflections. The second one is the signals reflected from the bottom of the horizontal stratum (copperbottom), simulating the primary reflections. The last one is the reverberations which are multiple P-wave reflections in the water. In this figure, the amplitudes of the primary reflections are smaller than those from reverberations. However, the amplitudes of the primary reflections are as large as the reverberations when the offsets are greater than $1450 \mathrm{~m}$ because the AGC effect allows the section to appear saturated. The reverberation waveform at the $250 \mathrm{~m}$ offset is over-saturated. It also reveals that the primary reflections and reverberations are mixed when the offsets are between 550 and $850 \mathrm{~m}$. The primary reflection and reverberation waveforms 
are broadened with the increase in offset. The reverberation waveforms are quite different from the primary reflection waveforms due to the wide-angle reflection. In the original section (before applying AGC, not shown in this paper), the amplitudes of the water-bottom reflections and the rever- berations decay faster for increasing offset. Conversely, the primary reflection amplitude decay is smooth.

In the travel time calculation, the source- and receivertransducer are considered as the point-source and pointreceiver. Since the velocities of the media and geometrical

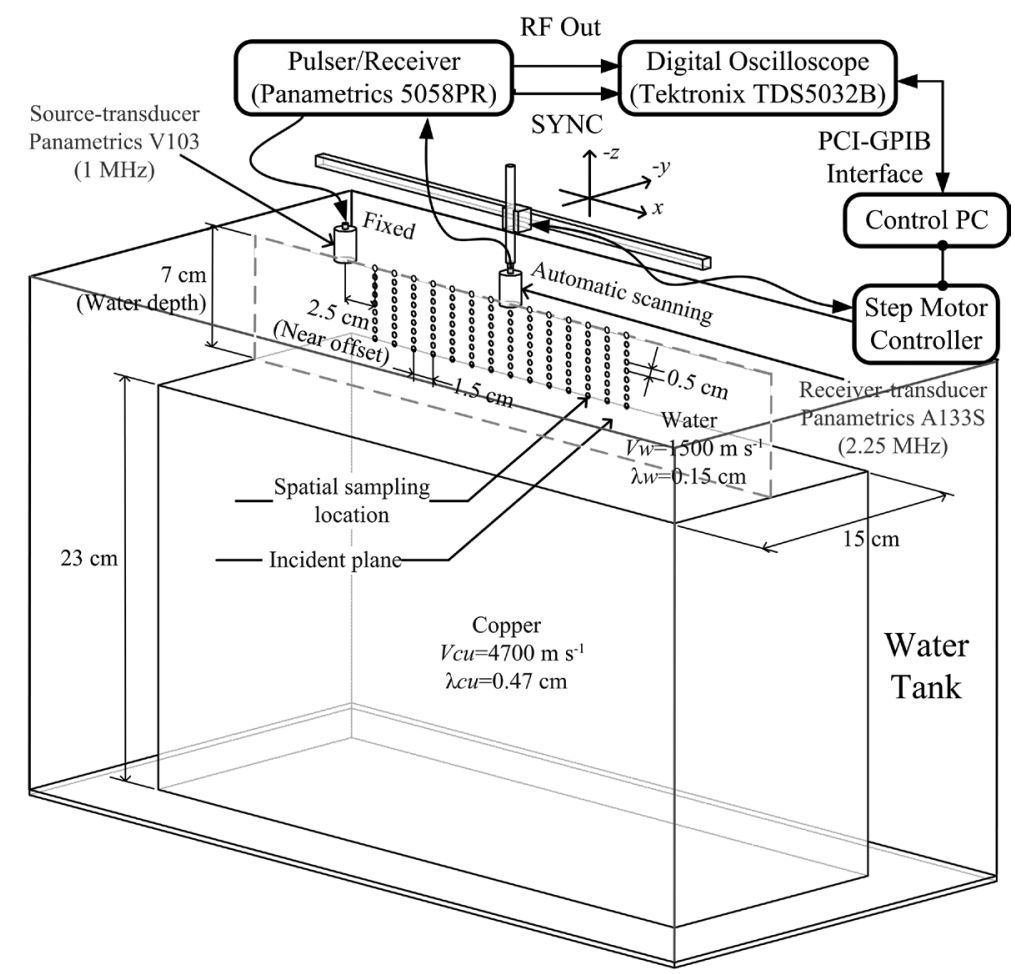

Fig. 4. Physical model, apparatus and recording geometry used in this study. The ultrasonic double-probe reflection technique is employed to simulate seismic data acquisition using multi-depth streamers.

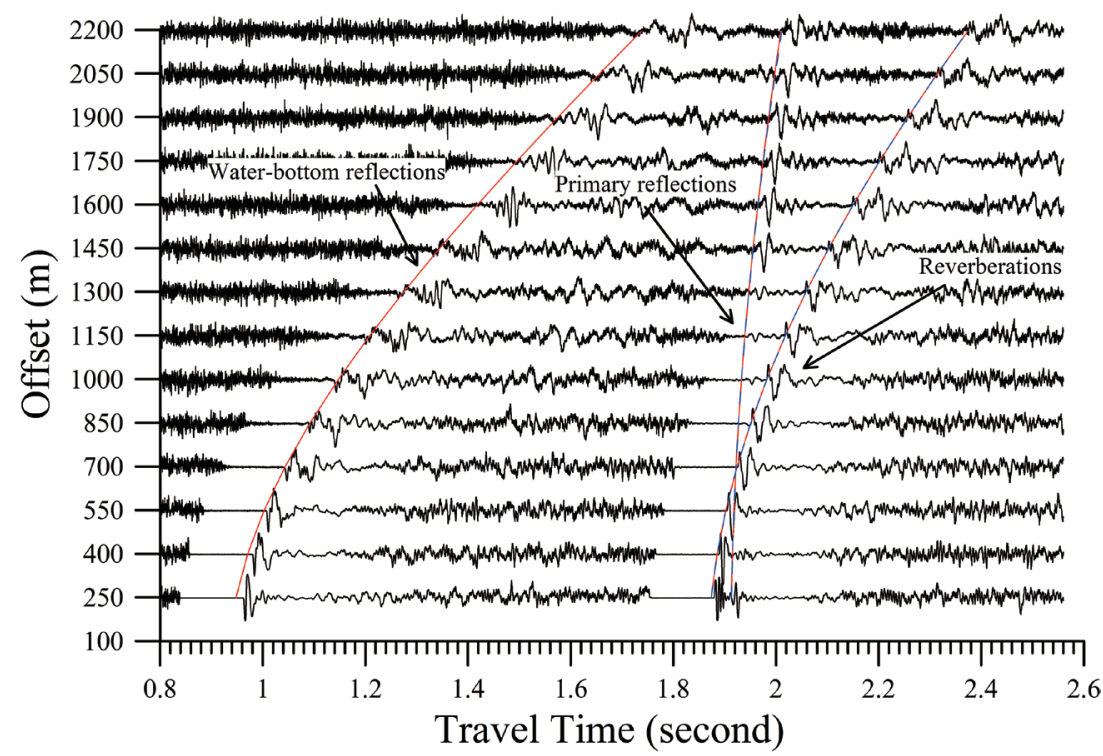

Fig. 5. The common-source horizontal-array gather received at the water surface. The red lines are the theoretical arrival times for the water-bottom reflections, the primary reflections, and the reverberations for a point-source and point-receiver assumption. The blue dashed lines are the arrival times for primary reflections and the reverberations calculated using the analytic Eqs. (2) and (6), respectively. 
relationship between the source-, receiver-transducer, and model are known, the theoretical arrival times of the waterbottom reflection, the primary reflection and the reverberation for a point-source and point-receiver assumption can be calculated using the seismic ray theory. They are shown by the red lines in Fig. 5. The offset-arrival time relationships of the water-bottom reflections and the reverberations are hyperbolic [Eqs. (6) and (7)]. For primary reflections, their offset-arrival time relationship resembles a hyperbolic curve [Eqs. (1) or (2)]. The arrival times of the primary reflections and the reverberations calculated by the Eqs. (2) and (6), respectively, are shown by the blue dashed lines. The red and blue dashed lines overlap each other and they fit the scaled physical model data very well. That reveals that Eqs. (2) and (6) work well for a common-source horizontalarray gather. The theoretical arrival times (based on a pointsource and point-receiver assumption) of the water-bottom reflections are earlier than the physical experiment results at near offsets ( 250 and $400 \mathrm{~m}$ ). The ultrasound wavelength in water is $1.5 \mathrm{~mm}$, which is much smaller than the sizes of the transducers used in this study. Therefore, the transducer is a large transducer related to the wavelength. This misfit is due to the finite size transducer effect on the ultrasound arrival time ( $\mathrm{Li}$ et al. 2000). In their experiments the ultrasound arrival times measured using the point-transducer precede those measured using the large-transducer at near offsets, which is consistent with our experiments. On the other hand, if the ultrasound propagation distance is much greater than the transducer size, for example the ultrasound measured at intermediate and far offsets, the transducer can be considered as a point-transducer. Therefore, the theoretical arrival times of the water-bottom reflections fit the scaled physical model data well for offsets greater than $400 \mathrm{~m}$.

A common-source vertical-array gather with a horizontal offset of $250 \mathrm{~m}$ is shown in Fig. 6. This can be considered a near offset configuration. The theoretical arrival times of the water-bottom reflections, the primary reflections and the reverberations are shown by the red lines. The arrival times of the primary reflections and the reverberations calculated using Eqs. (5) and (12), respectively, are denoted by the blue dashed lines. Again, the red and blue dashed lines overlap each other and they fit the scaled physical model data very well. That reveals that Eqs. (5) and (12) are correct for a common-source vertical-array gather. For this near offset acquisition, the theoretical arrival times of the water-bottom reflections precede the scaled physical model data due to the finite size transducer effect. The arrival times of three dominant events show approximately a linear relationship with the water depth. Based on Eqs. (5) and (12) the slopes of the primary reflections and the reverberations are $-6.67 \times 10^{-4}$ and $-6.64 \times 10^{-4} \mathrm{~s} \mathrm{~m}^{-1}$, respectively, which are almost the same.

For a far offset configuration, a common-source vertical-array gather with a horizontal offset of $1750 \mathrm{~m}$ is shown in Fig. 7. In this figure the theoretical arrival times (red lines) of the primary reflections, the reverberations and the water-bottom reflections fit the scaled physical model data well. Again, the arrival times (blue dashed lines) of the primary reflections and the reverberations calculated using Eqs. (5) and (12) coincide well with the theoretical arrival times except for some of the reverberations at deep depths (350 and $400 \mathrm{~m}$ ). The misfit of Eq. (12) at depth $400 \mathrm{~m}$ is small $(0.005 \mathrm{~s})$ which is about $0.3 \%$ of the arrival time. This is because Eq. (12) is based on a small spread and $h_{1}>z$ (the water depth is greater than receiver depth) assumption, thus for this far offset acquisition, the reverberation arrival times predicted by Eq. (12) at deep depths cannot perfectly match the assumption. The arrival times of three dominant events show approximately a linear relationship with the water depth. The slopes of red and blue dashed lines for the primary reflections are the same $\left(-6.63 \times 10^{-4} \mathrm{~s} \mathrm{~m}^{-1}\right)$. However, for the reverberations, they are $-5.53 \times 10^{-4}$ and $-5.65 \times 10^{-4} \mathrm{~s} \mathrm{~m}^{-1}$, respectively. The slope error of Eq. (12) is about $2 \%$, which is very small when compared with the stratum's velocity estimation in the field data and can be ignored in the seismic data processing. The primary reflection depth-arrival time relationship slopes are nearly the same for all common-source vertical-array gathers with different offsets because the propagation lengths of the primary reflections are much longer than the distance between streamers and offsets. However, the reverberation depth-arrival time relationship slopes increase with increasing offset [Eq. (12)]. Thus the difference in slopes between the primary reflections and the reverberations is proportional to the offset. Increasing the offset, therefore, leads to greater slope difference between them. The experimental results (Figs. 6 and 7) verify that the depth-travel time relationship derived in this study for the primary reflections and reverberations in a common-source vertical-array gather is correct.

Because marine seismic exploration is usually performed in shallow seas and the targets are normally much deeper than the water depth, the distance between streamers will be much smaller than the primary reflection propagation lengths. Therefore, the depth-arrival time relationship of the primary reflections for streamers at different depths could be deemed linear [Eq. (5)]. Because the reverberations are confined in the water layer, their depth-arrival time relationship is not as simple as that of primary reflections [Eq. (12)]. Hence, the data acquired using multi-depth streamers can be statically corrected to a specific water depth for primary reflections and the corrected data can then be stacked to enhance the primary reflections and suppress the reverberations.

\section{CONCLUSIONS}

The characteristics of reverberations acquired using multi-depth streamers were studied using ray path and physical modeling geometric analysis. The study results show that the primary reflection and reverberation waveforms are 


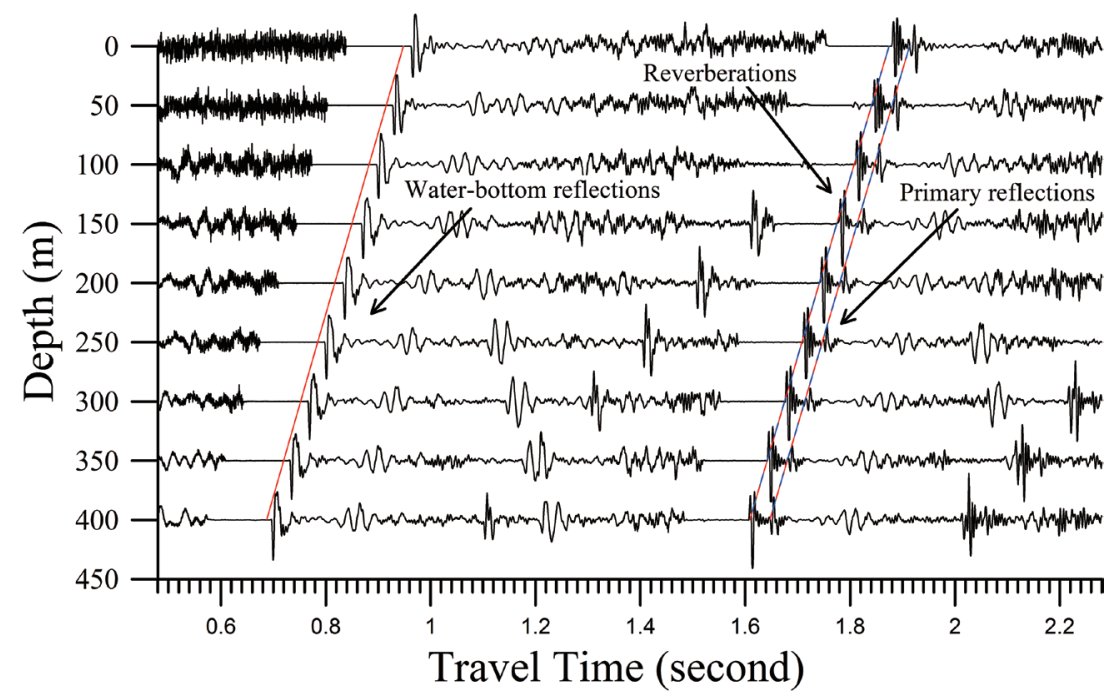

Fig. 6. The common-source vertical-array gather with a horizontal offset of $250 \mathrm{~m}$. The red lines are the theoretical arrival times for the water-bottom reflections, the primary reflections, and the reverberations for a point-source and point-receiver assumption. The blue dashed lines are the arrival times for the primary reflections and the reverberations calculated using the analytic Eqs. (5) and (12), respectively.

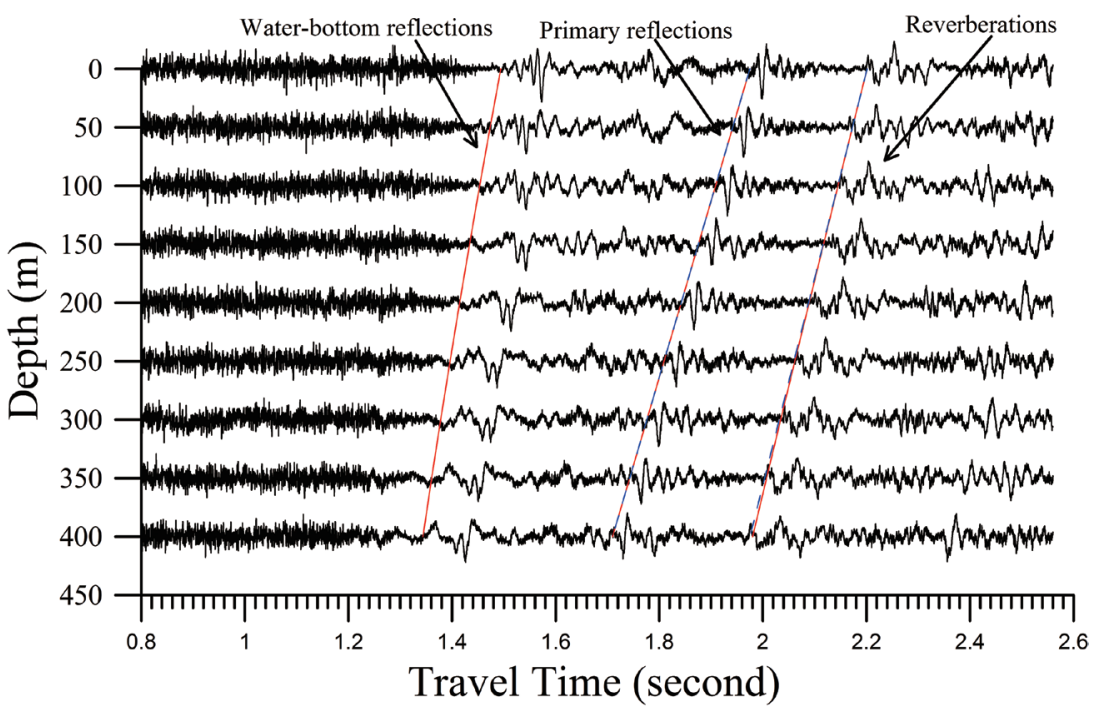

Fig. 7. The common-source vertical-array gather with a horizontal offset of $1750 \mathrm{~m}$. The red lines are the theoretical arrival times for the waterbottom reflections, the primary reflections, and the reverberations for a point-source and point-receiver assumption. The blue dashed lines are the arrival times for the primary reflections and the reverberations calculated using the analytic Eqs. (5) and (12), respectively.

broadened with increasing offset. The reverberation waveforms are quite different from primary reflection waveforms due to the wide-angle reflection.

Under shallow water and small spread approximation, new arrival time equations for primary reflections and reverberations were derived and fit the scaled physical model data very well. The depth-arrival time relationship of the primary reflections is linear in the common-source vertical-array gather. The depth-arrival time relationship slopes are nearly the same for different offsets. However, for reverberations, their depth-arrival time relationships in the common-source vertical-array gathers are also linear but they are different from the primary reflections. The depth-arrival time relationship slope of the reverberations increases with offsets.

Acknowledgements We are grateful to two anonymous reviewers for their helpful suggestions, which have largely improved our manuscript. This research was financially supported by the National Science Council under grant No. NSC98-2116-M194-006.

\section{REFERENCES}

Arvelo, J. I. and X. A. Zabal, 1997: Reverberation rejection 
via modeforming with a vertical line array. IEEE $J$. Ocean. Eng., 22, 541-547, doi: 10.1109/48.611146. [Link]

Backus, M. M., 1959: Water reverberations-Their nature and elimination. Geophysics, 24, 233-261, doi: 10.1190/1.1438579. [Link]

Canales,L. L. and M. L. Bell, 1996: Ghost attenuation using dual sensor cable data. SEG Technical Program Expanded Abstracts, 1591-1594, doi: 10.1190/1.1826427. [Link]

Ebrom, D. A. and J. A. McDonald, 1994: Seismic Physical Modeling, Society of Exploration Geophysicists, Tulsa, $519 \mathrm{pp}$.

Ferber, R., 2008: Adding streamers to an over/under configuration can improve imaging. World Oil, 229, 37-42.

Kleyn, A. H., 1982: Seismic Reflection Interpretation, Springer Netherlands, $270 \mathrm{pp}$.

Li, R., P. Okoye, and N. Uren, 2000: A study of the effects of transducer size on physical modeling experiments for recovering anisotropic elastic parameters. Geophys. Res.Lett.,27,3643-3646, doi: 10.1029/2000GL011401. [Link]

Moldoveanu, N., L. Combee, M. Egan, G. Hampson, L. Sydora, and W. Abriel, 2007: Over/under towed-streamer acquisition: A method to extend seismic bandwidth to both higher and lower frequencies. The Leading Edge,
26, 41-58, doi: 10.1190/1.2431831. [Link]

Monk, D. J., 1990: Wavefield separation of twin streamer data. First Break, 8, 96-104, doi: 10.3997/13652397.1990007. [Link]

Özdemir, A. K., P. Caprioli, A. Özbek, E. Kragh, and J. O. A. Robertsson, 2008: Optimized deghosting of over/ under towed-streamer data in the presence of noise. The Leading Edge, 27, 190-199, doi: 10.1190/1.2840366. [Link]

Robinson, E. A. and S. Treitel, 2000: Deconvolution for the elimination of ghost reflections and reverberations. Geophysical Signal Analysis, Society of Exploration Geophysicists, 251-267, doi: 10.1190/1.9781560802327. ch11. [Link]

Taner, M. T. and F. Koehler, 1969: Velocity spectradigital computer derivation and applications of velocity functions. Geophysics, 34, 859-881, doi: 10.1190/1.1440058. [Link]

Van Borselen, R., R. Hegge, T. Martin, S. Barnes, and P. Aaron, 2011: Enhanced demultiple by 3D SRME using dual-sensor measurements. The Leading Edge, 30, 920-926, doi: 10.1190/1.3626500. [Link]

Van Melle, F. A. and K. R. Weatherburn, 1953: Ghost reflections caused by energy initially reflected above the level of the shot. Geophysics, 18, 793-804, doi: 10.1190/1.1437929. [Link] 